\title{
Case Study 4: Technology Enhanced Workplace Learning
}

\author{
Michael Werkle, Manuel Schmidt, Diana Dikke, and Simon Schwantzer
}

\begin{abstract}
Within the ROLE research project it has been evaluated how personal learning environments (PLEs) perform in different surrounding conditions. Companies do often lag behind in the state-of-the-art developments from research especially in terms of Technology Enhanced Learning. Festo therefore observed on its internal test bed how PLEs can be implemented in business contexts and how to involve the learners in this process. Since there is already a broad variety of tools to organise and manage formal learning processes within companies the test bed didn't start by scratch either. The focus was thus on how to open up an existing learning management system (LMS) towards a PLE. During this process many experience from both learners as well as administrators, and training organisers have been gathered. One of the lessons learned is that a pure PLE doesn't fit the requirements on personnel development in business context, but certain PLE aspects can improve individual learning processes significantly. One showcase is the Festo LearningTube which was developed during ROLE. This is an example for the successful integration of user generated content into a corporate LMS.
\end{abstract}

Keywords Technology enhanced workplace learning • Personal Learning Management System • Evaluation • Test bed

\section{Challenges and Solutions in Technology Enhanced Workplace Learning}

The competitiveness of a company depends strongly on the skills and abilities of its managers and employees. The development of information and communication technologies (ICT) offers a wide range of tools and application options or even completely new forms of learning in this regard. Thanks to the development of the

M. Werkle $(\triangle) \cdot$ M. Schmidt

Festo Lernzentrum Saar GmbH, St.Ingbert-Rohrbach, Germany

e-mail:werk@de.festo.com; mans@de.festo.com

D. Dikke $\bullet$ S. Schwantzer

Information multimedia communication AG, Saarbrücken, Germany

e-mail: diana.dikke@im-c.de; Simon.Schwantzer@im-c.de

S. Kroop et al. (eds.), Responsive Open Learning Environments,

DOI 10.1007/978-3-319-02399-1_7 
Internet in particular, new multi-media learning scenarios and teaching methods increasingly complement, compete with and even partially replace classic classroom-based teaching.

While these are usually large companies in the field of formal learning which have been able to establish themselves in the area of classic e-learning or blended learning concepts on the further education market and in the field of staff development, technologies and learning scenarios for promoting informal learning in the workplace are given even less consideration or fail before they have got off the ground.

Why is this so? The idea of setting learning processes in motion or even optimising them simply by using new learning technologies for planning, performing and reflecting on further education courses, is just as harmful as the complete ignorance of such usage scenarios and potential. Not only technical requirements, but also media-didactic conception and design aimed at specific target groups are crucial for the efficient integration of learning technologies, which are usually web-based educational applications.

Teachers and learners within companies often lack media competence and the ability to apply self-regulated learning (SRL). Thus, train-the-trainer concepts for these skills are needed. Something that should not be underestimated is that innovative approaches often also fail due to the lack of willingness to change or poor implementation.

\section{Trends in Technology Enhanced Workplace Learning}

In the last years, the rapid changes in business environment set new requirements on the development of learning methods, as well as learning software and content. The experiences of the last years show that the curriculum-based learning approach, based on the assumption that the learner "consumes" knowledge in the form of predefined learning content, was continuously extended with SRL approaches based on the cognitive learning theory. This happened through the active involvement of the learner into the learning process, that is planning and defining the learning strategy, searching for appropriate methods, tools and materials, time management, reflection, and undertaking corrective actions. Such characteristics as adaptivity, responsiveness, and personalisation became, thus, mandatory for the modern learning software solutions.

Also, the penetration of mobile media, such as smartphones and tablets, into the Technology Enhanced Learning (TEL) branch caused significant changes in the conception and realisation of workplace learning activities. The possibility to use small software applications and pieces of content ("learning nuggets") became an indispensable component of virtual learning environments making the learning process time and place independent and optimised for mobile devices. Further, the "socialisation" of the web (e.g. the emergence of social networks, social sharing platforms, micro blogging, and so on) caused continuously increasing application 
of social media technology in education, using software tools supporting communication, content sharing, joint learning activities, and providing mutual feedback between the learners.

Thus, the following trends/challenges in terms of workplace learning have been observed:

- Increasing networking of the learners (collaborative learning/social learning)

- Creation of smaller, highly concentrated learning content (micro learning)

- Increasing importance of mobile learning ("bring your own devices")

- Individualisation and self-regulation of the learning processes (PLE approach)

- Videos as the learning medium with increasing potential

- Increasing importance of specific (experience-based) knowledge and user-generated content

\section{The ROLE Solution}

While learning, software met the requirement of becoming user-centred, flexible, and social, organisational structures needed more time to adapt to the new trends. The evaluations conducted within the scope of the ROLE Project showed that the vocational training and workplace learning providers appreciated the SRL approach and the idea of personalisation of the learning tools and content. On the other hand, they wanted to keep control over the learning environment, as well as tools and content provided to the end users. Further, it became obvious that the implementation of SRL in an organisation needed the development of specific competencies by the learners, as well as guidance through the learning process from its very beginning.

Thus, a learning software solution allowing combination of curriculum-based and SRL approaches, and providing both standard functionalities and content and personalised tools and materials has become necessary. To address this requirement, the ROLE Project developed a Personal Learning Management System (PLMS), which is an OpenSocial-based Learning Management System (LMS) combining functionalities of a LMS and a Personal Learning Environment (PLE) and allowing users to construct their virtual learning environment according to their learning history, goals, and preferences.

The following sections describe the main pedagogical and technical concepts underlying the development of the ROLE solution from the point of view of Festo, a test bed which actively contributed to the development, testing, evaluation, and application of the ROLE approach and technology. The Festo Showcase describes (a) the development and application of a learning video sharing portal (LearningTube), (b) the extension of the corporate LMS with additional webbased learning applications (widgets), c) the evaluation of the PLMS developed based on the corporate LMS and including external learning widgets. 


\section{Technology Enhanced Workplace Learning at Festo}

\section{The Learning Company Festo}

The Festo AG \&Co.KG was founded in 1925 by Gottlieb Stoll and Albert Fezer in Esslingen, Germany. Initially, it manufactured wood cutting tools and later diversified into the automation industry. Now Festo is a leading, worldwide supplier of automation technology with approximately 30,000 catalogue products, customised solutions, ready-to-install automation systems, and a matching range of before- and after-sales services. According to the Engineering \& Production on-demand concept, users can adapt these solutions to their individual needs, which actually increases the number of products offered exponentially. Furthermore, Festo is the performance leader in industrial training and education programs and offers a comprehensive range of learning systems for industrial training and educationfrom seminars, training and consultancy in 26 languages to e-learning and complete turnkey learning centres. Some 42,000 customers worldwide take part in Festo's seminars or are educated at the company's own training facilities. In 2012, the Festo Group had about 16,200 employees and a consolidated turnover of $€ 2$.2 billion. The 61 independent national companies serve customers in over 250 offices in 176 countries worldwide.

Festo AG \& Co. KG defines itself as a "learning company" which would like to constantly expand the knowledge and potential of its employees in order to encourage technical innovation and product development. Festo is a globally-oriented and independent family company with its headquarters in Esslingen. The largest production and logistics site is in St. Ingbert/Rohrbach, which is home to the cylinder production, the Customer Service Center and the Festo Learning Center.

\section{The Festo Lernzentrum Saar GmbH (Festo Learning Center)}

The Festo Learning Center was founded in 1994 and is located next to Festo's production plant and logistics centre in St. Ingbert/Rohrbach, Germany. As an accredited institution for advanced vocational training, the Festo Learning Center offers a wide range of personnel and organisational development programmes. The service portfolio comprises the whole spectrum of further education (seminars, training courses, the private technical school "Festo Technikum"), subsidy consulting, vocational retraining and qualification, industry consulting, and e-learning. The customers are international enterprises of all branches and institutions, as well as Festo employees and private persons.

Within the ROLE project, the Festo Learning Center aimed to define requirements of how to create a ROLE environment within a professional industry business. The Festo Learning Center wanted to demonstrate the use and benefit of ROLE technologies for the learners by addressing and supporting them in planning, 
realisation and evaluation of qualification measures with the help of the web-based services on the Festo Virtual Academy-Festo's global e-learning platform.

\section{The Virtual Academy}

The Festo Virtual Academy is the central LMS of Festo with a corporate internal focus. It is based on the software CLIX developed by IMC (see section "IMC and CLIX Learning Suite"). The LMS supports personnel development processes within the Festo organisation and offers strategic relevant online courses to the learners.

The Virtual Academy is open for each Festo employee worldwide for their personal further education by means of the lifelong learning approach. The LMS is accessible via the internet to facilitate SRL processes for the employees (learning independent of time and place). In addition to the self-learning offers, several blended-learning modules are available. The web-based trainings (WBTs) are designed according to didactic models in order to make their "consumption" as easy as possible for the learners.

The Virtual Academy has more than 9,000 users distributed all over the world, more than 80,000 logins p.a., more than 800 learning contents in different strategic learning categories with a total learning time of approximately 2,000 h. The contents are provided mostly in German, English and Spanish. For specific topics additional languages like Chinese, Japanese, Portuguese and Russian are also available.

In the ROLE project, the Virtual Academy was one of the five original test beds and addressed the issue of providing a responsive learning environment within further education activities in a company. This included not only continuous technical and media-didactical possibilities of a LMS and the content within, but also the technical possibilities beyond the LMS approach.

The application of ROLE at Festo led to better support of individual learning processes (in terms of self-regulation) and also better support of collaborative and social learning in a company. Therefore, informal learning increases its importance for the workplace learning and also the possibilities to record, share and save this expert knowledge within the company.

The following chapters describe the most important TEL-topics for the current and future developments of the Virtual Academy as well as the ROLE project with the Festo test bed, especially

- The initial situation

- The project vision

- The specific target group

- The characteristics of the business context

- The main organisation related challenges and the requirements

- The technical implementation

- The evaluation of the project results 


\section{Trends of Technology Enhanced Workplace Learning at Festo}

\section{Making Learning Environments More Personal}

The PLE approach is nothing particularly new, but is still a vital research topic in the TEL-community. PLE stands for a PLE, i.e. an individual composition of learning services that helps learners to plan and conduct their learning activities, and to reflect on the learning process and progress. Considering the fact that learners are often a very heterogeneous group (with different learning needs and goals, learning styles, individual learning experiences, knowledge, learning preferences or different job roles), the potential of the PLE approach is undisputed.

In 2007, Graham Attwell (2007) defined a PLE as being "comprised of all the different tools we use in our everyday life for learning". Considering this quote alone, one can be of the opinion, that in business contexts, especially in e-learning, every employee has already developed a PLE consisting of standard and companyspecific software as a digital toolbox. But far from it, at the moment the PLE approach is more theory than it is implemented in further education scenarios or personnel development processes in companies. It seems that further education is more and more lagging behind technical developments.

The main hurdles for the TEL implementation in companies are:

- At the moment, learning processes in companies are unilaterally strategic driven in a top down manner.

- Most of the learning processes are formal. The potential of informal learning and user generated content is not being exploited. The paradigm change of content towards the expert as author is not yet turned into practice in companies.

- Companies have already made big investments in learning technologies (perhaps in e-learning content or an LMS) and promote only these.

- Frequently there are a lot of existing internal learning tools in (bigger) companies, but these tools are often not synchronised. Therefore, the promotion of new learning tools across departments is very difficult.

Consequently, the Festo test bed examined in ROLE how the PLE approach can enhance learning opportunities for employees. The target was not to create a "pure" PLE, but rather to combine advantages of a PLE with an existing LMS. Thus, it was not a question of replacement or substitution of an existing, traditional LMS in a company but rather an approach that enabled the enhancement of their current delivery mechanisms. It was also a question of how an existing LMS can be enriched with new information and communications technologies that accordingly enhance the end-user (the learner's) experience. This will be explained in detail in the section "Implementation of the Personal Learning Management System". 


\section{Videos as the Learning Medium of the Future}

The power of the image is used on the Internet to disseminate information quickly and easily. Photos and graphics are omnipresent in the worldwide web today-be it for advertising or promotional purposes, to inform or simply to spread a bit of happiness. Today's technology makes it very easy for each and everyone of us to create pictures or videos and publish them on the Internet. Individual users already actively use video production and sharing to exchange experiences and knowledge. The trend towards video-based learning is being adopted by private companies and higher education.

Pioneers in this area include the Khan Academy ${ }^{1}$ with a thematic collection of over 4,100 instructional videos and Stanford University, which operates its own YouTube channel. ${ }^{2}$ However, the YouTube channel alone is not the impressive fact, but those 215,289 subscribers downloading over 55 million videos on demand, illustrates its reach. The private online academy Udacity, ${ }^{3}$ founded in 2012, provides similar figures. The philosophy here is "Learn. Think. Do.- - Higher Education for Free". The first two free video-based online courses alone reached 90,000 participants (Lewin 2012).

The key to the success of these video tutorials is the didactic simplification through visual representation. Wherever one previously had to describe a subject in words, written or verbally, or how complex product drawings had to be created, today it is simple to visualise information using moving, narrated images. This usually saves the knowledge carriers and producers of video tutorials more than just time in comparison to creating professional teaching materials or specialist articles. The creator of a video tutorial is able to use the sound and video track to give his "knowledge" not only a personal note but also a kind of personal signature.

The knowledge content is closely connected to the knowledge carrier and these are not as easy to separate as in the case of pure text-based content. Thus, statements, opinions and experiences can be expressed personally through interviews and the interviewed person "signs" it with his name. Ultimately, the web video medium suites often better to meet the need for communication and selfexpression than "pure" text. From the learner's point of view, particular application or process knowledge is taken in more easily via pictures and transferred to "real life" than when if it is read from texts. The recipients also use their auditory and visual sensory paths to process information, which helps to anchor it in the memory.

Festo has recognised this trend towards instructional videos. It developed an award-winning tool, the LearningTube and Recorder, as part of the ROLE research project. The LearningTube provides all Festo employees with a simple way of creating video tutorials and sharing them with colleagues in the company in order to document best practices arising in everyday work and make them available for

\footnotetext{
${ }^{1}$ http://www.khanacademy.org/about

${ }^{2}$ http://www.youtube.com/user/StanfordUniversity

${ }^{3}$ https://www.udacity.com/us
} 
colleagues, successors, and new employees. The innovative aspect of that is the combination of the LMS with easy-to-use authoring software which allows content owners as well as every learner to instantly create learning content on their own. Compared to traditional LMS that only had "time-consuming" text-based authoring tools included, the screen recording capabilities of the LearningTube make it much easier for the authors to capture their knowledge and transform it into learning content. The reason for the perceived simplification is that experts can easily reuse the tools and media they use in their everyday life to transport the information such as PowerPoint slides or recording the software they need to explain. Thus they do not need to develop new skills, the system is built on the skill-set the experts already have.

\section{The ROLE Project and the Festo Test Bed}

\section{Project Vision}

\section{Learning in the Virtual Academy Before ROLE}

The learning processes at Festo can be described as self-controlled by the learners (employees). The users browse offered learning programmes in the catalogue of the Virtual Academy and select those they need. During the learning process itself, the learners can decide by themselves what they would like to learn and which parts of the web-based training content they would like to skip. Since the platform is available online, the learners can even access the Virtual Academy from home and participate in trainings on a voluntary basis. The learners are free to define and plan what and when to learn, and how frequently to work on the web-based training offered in the catalogue.

The vision of the ROLE project was to improve especially the aspects "responsive" and "open" of the already existing learning environment of Festo - the Virtual Academy as the central LMS of the company. From Festo's point of view the main targets were to improve existing learning systems according to:

- Openness and adaptivity

- Communication with other learners

- Facilitation of collaboration and peer-assisted learning

- Switch collaborated and individual work

- Exploring ways of benefiting from the experience acquired in a company

- Best practice sharing 


\section{Challenges and Requirement Analysis of the Test Bed}

The Festo Learning Center aims to meet the requirements of learning environments within a business context. To achieve this aim, Festo effectively opened up its platforms and tools to "mix and match" and be interoperable through the use of ROLE tools and technologies.

\section{Organisation Related Challenges and Requirements}

Since the Festo Virtual Academy is effectively a corporate learning environment, some special surrounding conditions have to be considered within the ROLE process. These special "challenges" depend on the fact that a corporate LMS has one central main function: the further development of personnel in the company.

Issues relating to these surrounding conditions are, for example:

- The uncertain scope of openness of a corporate learning environment. How wide can a corporate learning environment be opened up?

- The "job role" of the learner has to be in the focus of all learning processes

- Knowledge sharing is harder to realise in the job context than in non-working life

- Understanding learning processes during daily work and after work

- System restrictions and data security

\section{Target Group Related Challenges and Requirements}

The Festo test bed is focused on LMS users and especially LMS users in a company. This target group has special needs and the surrounding conditions in companies are not as flexible as those predominant, for example, in universities. In contrast to students, business learners are a very heterogeneous target group with big age differences (from 16 to 65), different educational backgrounds and previous knowledge, job-roles, learning requirements, learning preferences and learning goals.

Further, the learners in business environments have primarily to fulfil their job role and learning is mostly to support them in doing so. Due to high workloads, it is often hard to learn on the job or in other words, there is no or just little time for learning available. It is often not so easy to disengage workers from their daily practices. Thus, learners at the workplace need to be supported systematically, not only with new TEL solutions, but also with their development goals, the working and learning conditions in general and their work life balance. 


\section{User Related Challenges and Requirements}

There is one more aspect to be considered, which is related to the personal requirements of the learners. The learners need a set of specific skills, the so-called "self-regulated learning" (SRL) skill-set. These are skills the learners must have to be able to successfully plan, conduct, and evaluate their learning activities. Some of these skills might be new to some learners, whereas others might be present and used already, but the awareness about that fact is still missing.

To address this requirement, Festo initiated the "Fit for Self-Regulated Learning" initiative in the ROLE project. The plan was to implement various SRL learning modules in the offered ROLE service. Some of the modules should explicitly show that they support learners in getting SRL known as a method. Others would be implicitly woven into ROLE services to make their impact on SRL visible, for example within wizards.

Putting the idea behind "Fit for self-regulated learning" in a nutshell, one can state that SRL should improve the learning outcomes. This is realised by offering the learners SRL as a manual. This would not be a technical description of the platform, but rather a manual for learners that shows how to learn effectively and successfully in the ROLE environment with the help of SRL.

Festo considered the benefit of the approach to be very high and therefore decided to personalise learning through ROLE and made learning more demandand service-oriented for the users of the Festo Virtual Academy.

\section{Implementation of the Personal Learning Management System}

\section{IMC and CLIX Learning Suite}

IMC is currently Europe's largest learning management solution provider that was established in 1997 by Prof. Dr. h.c. August-Wilhelm Scheer as a spin-off of Saarland University. IMC offers a comprehensive portfolio of e-learning-related products, supporting all business processes in training and education. IMC also provides professional services covering the whole value chain of content design and production as well as consulting and managed learning services helping clients to (re)organise their learning processes and to select, implement, adapt and integrate suitable software systems and technologies.

IMC's product portfolio includes professional authoring and content recording tools (Content Studio, LECTURNITY), a Business Process Guidance System LIVECONTEXT, as well as a Learning and Talent Management System CLIX supporting HR- and Personnel Development processes at organisations. CLIX contains two main modules: the Learning Suite, which is a LMS in the classic sense, and the Talent Suite supporting Talent Management processes, such as 
Talent Identification, Competency Management, Career and Succession Planning, Performance Management, etc.

In scope of the ROLE Project, IMC used CLIX Learning Suite to implement the concept of the Personal LMS. CLIX Learning Suite in its basic configuration includes such functionalities as Organisation Management, Process Management, Content Management, Testing and Assessment, Resource and Capacity Management, Report and Compliance, Evaluation, and some others. The Social Learning module contains communication tools, such as chats, communities, wikis, and forums allowing making use of the synergy effects of collaborative learning.

In order to increase personalisation of the system and to extend collaborative functionalities, CLIX Learning Suite has been integrated in scope of the ROLE Project with an OpenSocial PLE, thus, constituting a so called Personal LMS. The PLMS aggregates learning resources and applications available in the web and selected by the users. Its structure aligns phases of the SRL process and assists the users in planning of their learning activities, search for learning content and tools, training and testing, as well as in reflection and evaluation of the learning progress.

Addressing the increasing need in acceleration of the knowledge acquisition due to rapidly changing organisational and technical conditions at organisations, and in personalisation of the learning process increasing employees' motivation and effectiveness of the training measures, an adaptable learning environment supporting workplace learning had to be developed. The identified requirements from the user side were: firstly, for the learner's point of view, to make the system customisable in terms of tools and content according to the current learning needs, and secondly, from organisational point of view, to keep control over the learning environment to ensure transparency of the learning process and the achievement of planned learning results.

\section{From LMS to Personal Learning Management System}

The early development of LMS is aimed at coordinating the learning processes in organisations in terms of training measures participant and content management, including creation of course curricula, adding learning materials and tests, as well as providing meta-data such as course dates and place, procedure of entering the course, course completion and certification. Over the last years the LMS developed in the direction of the Human Resource Management Systems (HRMS) providing functionalities for personnel assessment, competency management, learning and development, as well as succession planning. While increasing the planning and organisation capacity of the LMS, the learning process supporting infrastructure has not changed much.

The new learning approaches, such as SRL, collaborative and social learning have been realised in PLEs allowing the users to construct their learning environments themselves by selecting learning tools and content in a way supporting acquisition of the desired knowledge. In scope of the ROLE project, a PLE is a 
web-based infrastructure, where the users can access, aggregate and manipulate learning applications and resources of their preference, as well as communicate with other users sharing experiences and collaborating on projects (Overton 2009). Importantly, the PLE uses web widgets, which are small web-based software applications, to support particular learning and teaching goals or training of some specific skills.

In order to create a software solution supporting both approaches, an OpenSocial PLE has been integrated into LMS combining functionalities of both systems. Thus, the PLMS provides instructions and pre-defined learning materials allowing the users to complete learning courses as usual, but also it ensures the learning process autonomy offering personalised learning spaces, in which the users can add and use additional applications and resources. Further, the PLMS allows browsing additional learning content directly out of the system with help of the Media Search Widget, thus, being more open than classic LMS. Also, it offers guidance through the SRL process and support to its each phase, which are described in the sections below.

\section{Psycho-Pedagogical Integration Model and Personalised Learning Spaces}

The OpenSocial directory of the PLMS is structured according to the phases of the SRL process described in the Psycho-Pedagogical Integration Model (PPIM) developed in the ROLE Project (Fruhmann et al. 2010). This model identifies four main steps:

1. Plan: This phase includes definition of the learning strategy, learning goals, actions to be taken to achieve these goals, as well as preferences in the sense of tools and types of content that will be used.

2. Search: At this step the learner searches for learning resources and tools within the learning environment and outside of it. Here, the user may get recommendations from tutors and peers, but also use recommendation systems to find appropriate resources.

3. Learn: Learning includes studying of the selected tools and materials, attaining skills, training and testing, as well as assessment by tutors and self-assessment.

4. Reflect: This phase implies gathering feedback from different sources and selfevaluation, as well as reflection on the learning process and achievements in order to evaluate the usefulness of the learning strategy and particular actions and their correction if needed.

These phases are summarised as "plan-search-learn-reflect" loop (see Fig. 1 (Mödritscher and Nussbaumer 2012).

The OpenSocial directory of the PLMS is divided into four learning spaces corresponding to these four steps. In order to provide necessary guidance to the 


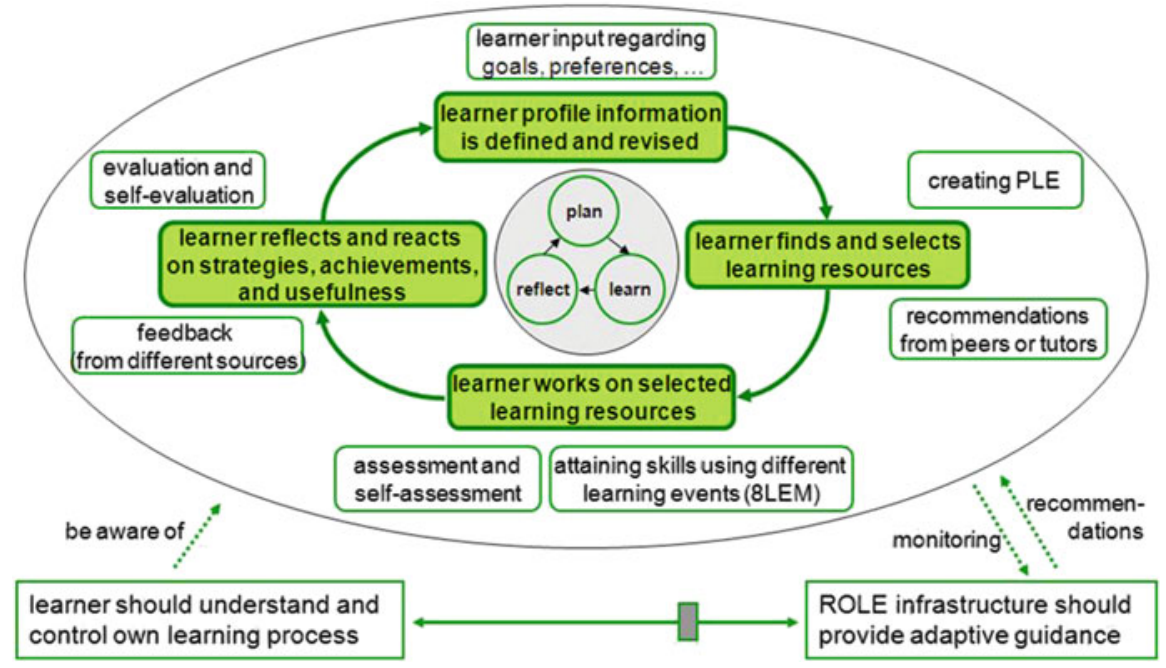

Fig. 1 Psycho-pedagogical integration model

users, each learning space is populated with pre-defined learning widgets. These are Open Source tools that were developed by the ROLE project or found in the web (all rights reserved) and reviewed concerning their suitability to support one or more of the learning phases. Further, the PLMS contains a list of additional tools. Thus, the users may use pre-defined applications and/or supportive tools from the list arranging them in the learning spaces. This allows an efficient integration of external tools into the system respecting the interests of both the learners and the organisation (Fig. 2).

Besides learning applications, each learning space contains an introductory video to the respective phase of the SRL learning process and a Wizard Widget containing supporting questions and hints to assist the user in getting started with using the system. The user acceptance evaluation showed that the availability of these assisting tools was highly appreciated by the users (see Evaluation section). Thus, the PLMS aims at providing as much guidance as necessary, and as much assistance as possible (Schanda et al. 2012). The development of the PLMS and its technical implementation focused on the personalisation, adaptivity, and userfriendliness of the PLMS making it responsive to the needs and preferences of the users.

\section{Technical Implementation: OpenSocial LMS}

In scope of the technical implementation, two major aspects were identified in order to extend the CLIX Learning Suite to be used as PLMS: on the one hand, a mechanism was required to enable the integration of external components into 


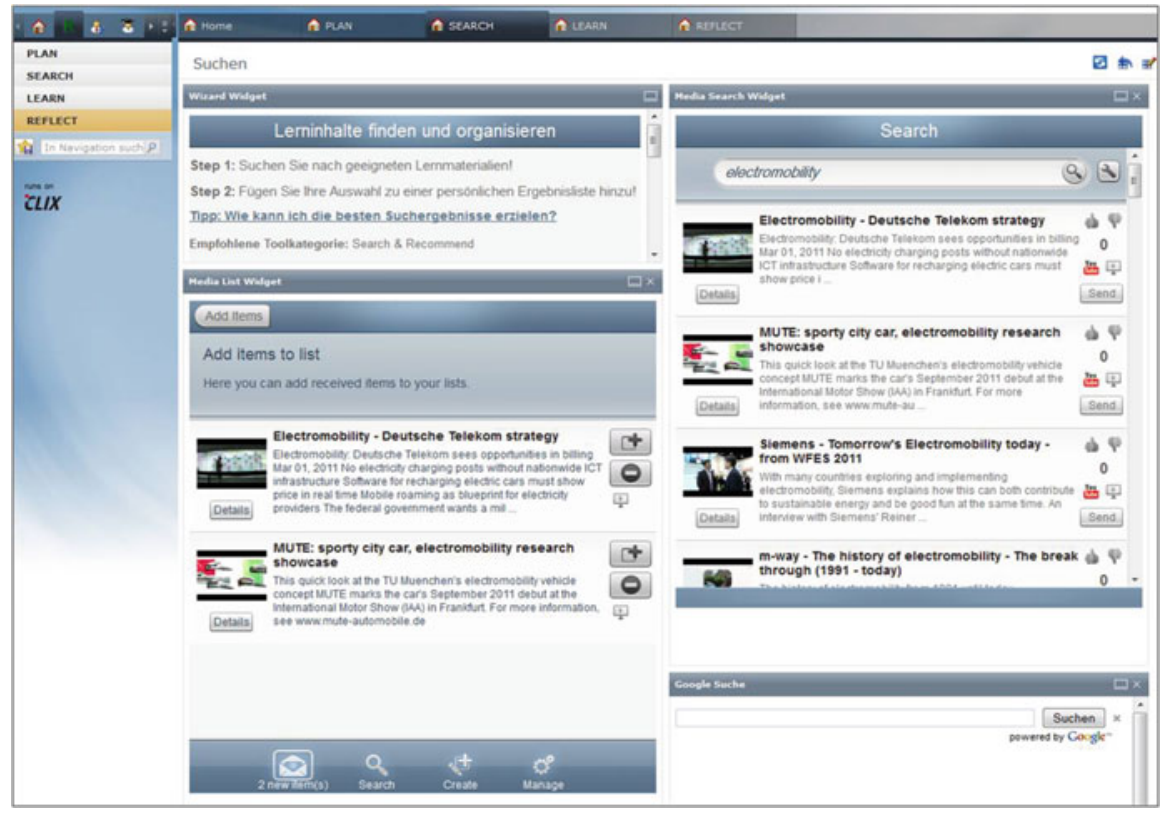

Fig. 2 ROLE PLMS search learning space

the CLIX portal. These components had to be implemented using a standardised technology for the following reasons:

- In order to separate the development processes and technologies for components of PLMS from those of CLIX.

- To enable external developers to create components without technical knowledge of the environment they are embedded in.

- In order to allow integration and re-use of existing components, e.g. components which are provided in the repository.

On the other hand, an interface was required to exchange social information between PLMS components, CLIX as a hosting environment, and external services.

Taking those requirements into consideration, the decision was made to use OpenSocial $^{4}$ as an open cross-platform standard specifying a REST-API to exchange social information. Having the API specified, the technology stack was completed by supporting the Google Gadget specification ${ }^{5}$ to create components, further on called widgets. Widgets are applications designed to only take small parts of a screen and make use of web technologies (HTML, CSS, and JavaScript). For the communication between the widget and the environment, a JavaScript API was provided, which also included the interfaces of the OpenSocial API.

\footnotetext{
${ }^{4}$ http://opensocial.org

${ }^{5}$ https://developers.google.com/gadgets/docs/spec
} 
The implementation of the OpenSocial API and Google Gadget specification was based on the Apache Shindig project. ${ }^{6}$ Apache Shindig was realised as a standalone web application, which was connected over a dedicated web service interface with the CLIX Learning Suite. This architecture allowed separated maintenance processes for both platforms. Shindig took two roles within the PLMS: as rendering server for widgets and as OpenSocial API provider. CLIX acted as data provider and environment for embedding the widgets rendered by Shindig.

At the next step, an integration point for the widgets inside the CLIX portal had to be found. As the PLE structure does not necessarily depend on a concrete course, the integration was realised using dashboard pages. Dashboard pages were designed to create a mash-up of panels showing user-centred information (e.g. a course list, news, social media updates, etc.) and therefore built a capable environment for widgets. The implementation aimed to provide a seamless integration with existing dashboard features. A new panel type was introduced and each instance of this panel represented a single widget.

Platform administrators can select which widget panels are available on a dashboard page and can pre-configure the widgets. They can choose if a widget has a system-wide or a user-specific configuration. In the latter case, the users have their own configuration interface for the widget directly integrated in the dashboard page.

The final layout and content of a dashboard page allow nearly any degree of personal individualisation. Whilst the pool of available widgets is previously defined, their visibility, application, and position can be made customisable for the individual user. The range of self-controlling is therefore very large; both settings of fully pre-configured widget mash-ups and open-space solutions, where users combine widgets starting from a blank page, are possible. Learning processes can also be supported by combining multiple dashboard pages, covering either different steps in a learning process or different topics addressed by specific pages.

\section{Concept and Evaluation of the PLMS at Festo}

\section{The Virtual Academy Case study}

This case study reports on how the ROLE environment can be open to a mix of internal learning applications alongside external ones. This is regarded as a key success factor for project ideas that emerge from developments like ROLE to influence the promotion of further education in companies and meets the overarching premise of this case study namely that it demonstrates "an internal learning opportunity in a company" environment. In essence, this section, thus, presents an

\footnotetext{
${ }^{6}$ http://shindig.apache.org/
} 
evaluation of a case study relating to the Festo Virtual Academy and demonstrates how internal learning opportunities can be improved in a company.

This case study therefore sets out to address the issue of providing a more responsive learning environment within the further education activities of Festo. As previously indicated, to achieve this aim, Festo opened up its e-learning platform to be interoperable and open for mixing and matching with ROLE tools. This combined approach was called a "Personal Learning Management System" (PLMS), where the PLMS was comprised of the LMS and PLE together, hence when combined resulting in the acronym PLMS as technically described in section "Implementation of the Personal Learning Management System".

The actual implementation of this approach, i.e. the degree to which these PLE enrichments are embedded, ultimately depended on both company-specific requirements and on the individual learning preferences, as well as the anticipated learning experiences of individual learners. In general terms, however, the main targets of the PLMS approach were:

- Simplified access and advanced search of relevant content and learning materials.

- Support and improvement of the planning of learning, incorporating the reflection phases of the learning activities.

- Enabling learning motivation and promotion of SRL as well as different forms of cooperative learning.

To achieve this improvement, Festo supported ideas and the development of prototypical widgets and tools, which can enhance the learning processes in such corporate learning environments. Initially, the first item to be addressed during the implementation of the ROLE approach was to improve the "Openness" of the system.

At the first step, a federated search widget was developed together with two other ROLE partners, namely IMC and KU Leuven. The idea of the widget was to enable a more focused search facility in the Festo LMS. It was developed to search learning content in several external online resources and feed results back directly to the learner within the Festo LMS. A media-list widget, that was interoperable with the federated search widget, was developed too. This allowed users to create media lists out of the resources found with the search widget. Both widgets are featured in the Festo Virtual Academy.

At the second step, another perspective of openness considered in the context of this case study was the integration of user generated content as well as encouraging the possibility for learners to produce content on their own. To achieve these outcomes, a commercial screen recorder facility was embedded as a widget, which enabled learners to create their own videos. This widget bundle was given the names of "LearningTube" and "Recorder", and together both widgets provided all learners, trainers and experts with appropriate support for exchanging and communicating training content on a daily basis.

In this case the Recorder allowed users to create a video, which could then be uploaded to the LearningTube and shared with Festo colleagues worldwide. Both 


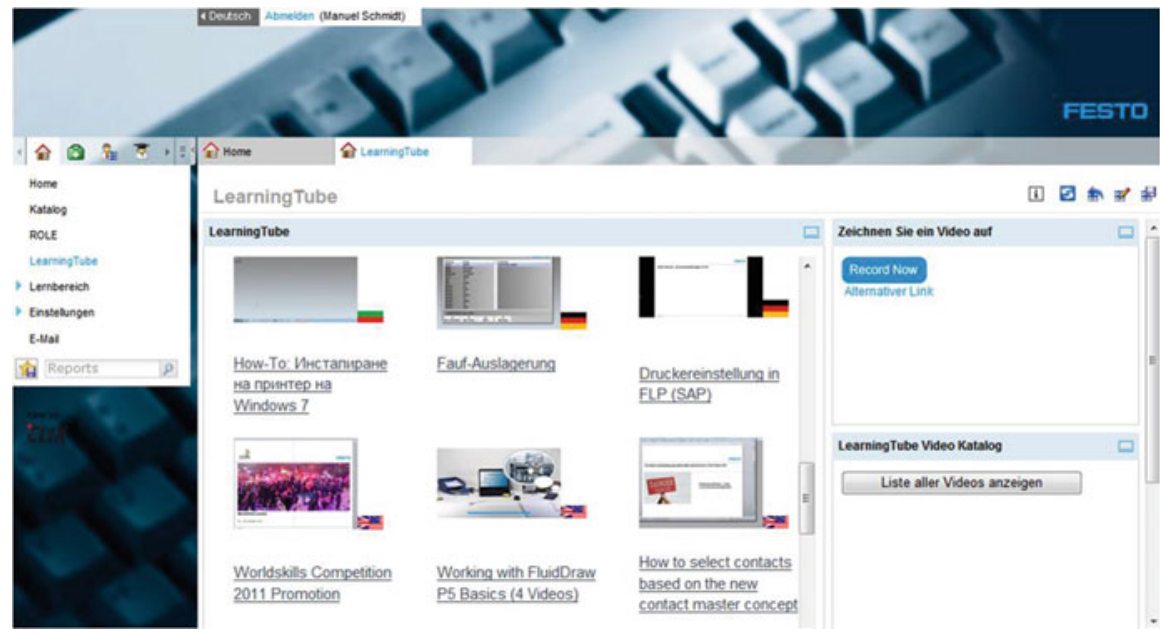

Fig. 3 The Festo LearningTube

tools supported bite-sized learning and ensured that content could be published and distributed quickly. This style of rapid e-learning, therefore, allowed users to enhance their presentations with the addition of a voiceover and optional webcam video. The Recorder widget also provided help and support for creating resulting screenshots, enabling users to add their own commentary to PowerPoint presentations or even enabling them to emulate software simulations.

The LearningTube widget bundle was acknowledged by the ComeniusEduMedia organisation, which gave an award for the practical application of educational, thematic and design excellence in educational media. Festo was the only industrial enterprise to receive such an award. Equally, the widget has offered to Festo employees around the globe, an interactive educational video channel that has also become a valuable learning tool. As a result of this implementation, there have been over 220 video uploads by Festo employees since July 2011, consisting mostly of screencasts and recorded presentations. These videos have been accessed over 15,000 times to date (April 2013) (Fig. 3).

\section{Evaluation}

The concept used to evaluate the ROLE solution at Festo consisted of a combination of questionnaires, selected expert interviews, a focus group for requirement gathering purposes, a taskforce observation and interviews by project members. These various elements ensured that there were standardised frameworks for evaluation and also personal contacts to offer possibilities to clarify confusing questions and allow a little more depth. 
During the project, Festo carried out two main evaluation loops. To this end, Festo founded a small focus group of 26 colleagues to test and evaluate the ROLE technologies in the Virtual Academy from the learner's perspective. Qualitative data about the usefulness, usability, look and feel, the innovativeness, the relevance, the clarity and the improvement potential of the presented ROLE technologies were collected. Therefore, the first evaluation loop was conducted by a specific questionnaire, whereas the main focus of the second evaluation loop was to observe how the test persons work with the learning technology.

The use of the focus group meant that although the evaluation was not on a large scale, the results were of high quality. This was due to the expert knowledge, the aforementioned method mix and the possibility to read between the lines and to receive more detailed feedback.

\section{The First Evaluation Loop}

As described, the first step was to implement two widgets in the existing Festo Virtual Academy LMS, thus, enriching it with appropriate PLE elements. The first evaluation loop consisted of a questionnaire about the developed media search and the media list widgets. The evaluation of these widgets was not possible with the existing standard questionnaire of the virtual academy. Therefore, a new questionnaire, asking about personal information, preferred forms of further education, daily use of the internet, affinity to Web 2.0, benefit of web 2.0 and the described widgets, was created. This questionnaire was emailed to the members of the previously described focus group; a screencast introducing the ROLE project, the ROLE approach, and the developed widgets was attached.

\section{Results}

The response rate of $61.5 \%$ (16 of 26) was not as high as expected, but it should be kept in mind that the members of the focus group had to give priority to their normal jobs and the effort required in the different evaluation loops had to be reasonable. The focus group supported the Festo project team on a voluntary basis partly in their leisure time. So their resources and feedback were important, but also very limited. All responses to the questionnaire in this case study regarding the look \& feel, usability and perceived usefulness were very positive concerning the applied ROLE approach in the business context. It showed that $63 \%$ of the users liked the look \& feel as well the usability.

Nineteen percent said that that the look \& feel should be improved and $13 \%$ stated that the usability should be improved. Regarding the quality of the search results, the performance and the fun factor of the widgets, $38 \%$ of the users said these issues were in need of improvement. Forty-four percent rated the quality of the search results as good, the performance got a good grade from $50 \%$ of the users, the fun factor was rated as good by $38 \%$ and even as outstanding by $13 \%$ (Fig. 4). 


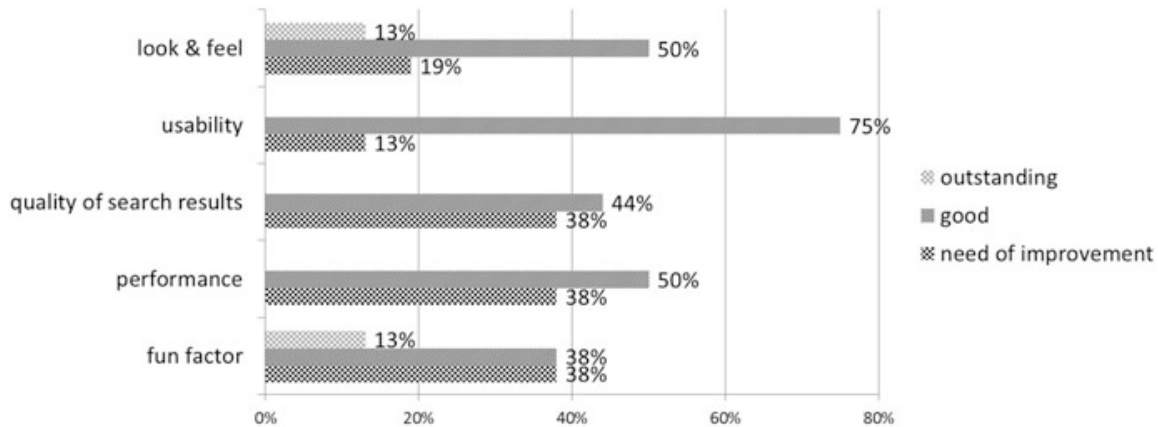

Fig. 4 The first impression of the prototypes

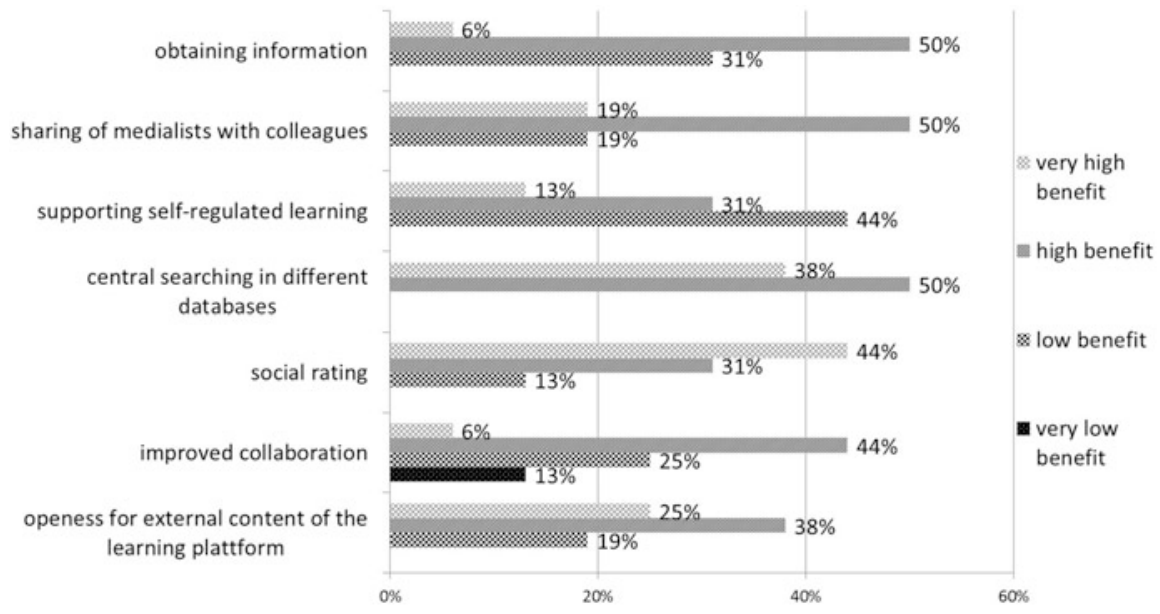

Fig. 5 Perceived usefulness of the prototypes

Evaluation of the benefits of the prototypes showed that most of the users saw a high or very high benefit of the offered tools. The highlights regarding the benefits were in this case the federated search feature over several knowledge resources. Fifty percent stated a benefit and as many as $38 \%$ stated a high benefit. For the rating feature $31 \%$ stated a benefit and as many as $44 \%$ stated a high benefit. The good overall impression of the evaluation reflects especially in the recommendation value - in total $88 \%$ of the test users would recommend the tools to their colleagues. Last but not least, perceived usefulness and effectiveness was evaluated with the question "do you think the offered services will help you to work more effectively in your job then at present?" Thirty-one percent fully agreed, $50 \%$ agreed and only $6 \%$ denied that they would be more effective when working with these tools. This was a really good result for this early prototype evaluation from Festo's point of view (Fig. 5). 


\section{The Second Evaluation Loop}

The subject of the second evaluation loop was the PLMS prototype, which was developed with the goal of integrating the approaches of the LMSs and the PLEs, and thus of supporting the user during SRL. The evaluation was conducted on a PLMS prototype, which was integrated into an IMC test environment.

The acceptance of the learning environment was and is an essential aspect in this regard. In particular, if an introduction of training offers, which will be used on a voluntary basis, is concerned, it is important for the training provider to determine the acceptability extend of the learners. Thus, the objective of the evaluation was to find out how well the PLMS was accepted by the employees, and which measures could be implemented to further increase its acceptance.

The following usability factors were important:

- The selection and addition of widgets, and navigation learning spaces

- The use of resources, such as wizard widgets and tutorials

- Acceptance of the PLMS

- Handling of the PLMS

- Comparisons with conventional training media and forms of learning

Therefore, several methods were used in order to evaluate the PLMS. Observation was conducted on the one hand, and the so-called "think aloud" or "question asking" method was used on the other. In addition, the questionnaire entitled "Perceived Usefulness and Ease of Use" was used in this evaluation supplementing data obtained by means of observation and the "think aloud method" ("TAM"). For reasons of comparability, the questionnaire was developed within the framework of the ROLE project, which was intended to ascertain usefulness and user-friendliness of the project results as perceived by the learner. It was also used in other test beds.

The "TAM" was used as a supplementary method in addition to observation. In particular, this method was used for examinations of the user interface because it is especially well suited for detecting problems of this sort. With the use of "TAM", the thought processes of the learners were investigated while they were dealing with the PLMS.

Test persons were prompted to describe their actions and thoughts out-loud during interaction with the learning environment. In this way, the test persons were not only able to address any problems they were experiencing with the user interface, they could also explain them and, as a result, could reveal design defects. Furthermore, the test persons expressed thoughts of satisfaction, enthusiasm or motivation when commenting on their actions. Thus, data compiled by means of "TAM" provided in-depth knowledge, not only regarding the actions of the test persons, but also about their attitudes as well. If applicable, information about their experiences gained in dealing with the software, could also be gathered.

The "TAM" was used in a slightly modified form, which was more comparable to an interview technique, by means of which the study director asked the user targeted questions regarding comprehension, the sequence of the learning activities, 
etc. This counteracted the danger that the test person talked less and less while working with the learning environment, and no longer remembered to comment on his actions out loud. This problem might have occurred because working with the PLMS represented a very new and complex task, for which the user required a large portion of his cognitive capacity.

The evaluation supervisor spoke with the test persons or helped them work through the learning steps in the PLMS, if requested to do so. However, this influenced the experiences, the attitudes and the actions of the learners. This influencing factor was taken into consideration in the evaluation of the results. As a rule, "TAM" was conducted with help of audio or video recordings. Instead of a reconstructive form of data collection, "TAM" made use of so-called registrative data conservation. This simplified evaluation and assured the reliability and validity of the compiled data. "TAM", thus, effectively compensated the weaknesses of participative observation.

\section{Evaluation Procedure}

During the evaluation phase 11 interviews were conducted with employees of the Festo Learning Centre. The employees came from different departments and had different educational backgrounds. The evaluation was allotted a duration of 4560 min per test person. The users were requested to complete a task in the PLMS to this end. Subsequently, the test persons evaluated the PLMS with regard to acceptance, system performance, required effort and user-friendliness, as well as use of and satisfaction with the application assistance.

The survey "Perceived Usefulness and Ease of Use" consisted of two parts. Those questions which were considered as learning premise, namely those regarding age, sex and TEL experiences were presented before the PLMS test. The other part of the survey dealing with the evaluation of the product, in this case the PLMS, was conducted after the PLMS test.

The evaluation supervisor was available while the questionnaire was being filled out, and was able to help the test persons with any questions or uncertainties. However, the test persons were initially only asked to respond to the closed questions. Then the observation started, during which time the observer took notes. If it was needed and time allowed it, the observer went deeper with the open question section in order to clear up any unanswered points.

\section{Results}

The evaluation of the questionnaire entitled "Perceived Usefulness and Ease of Use", provided the following summary: Eighty-one percent of test persons rated the learning environment PLMS as useful. Fifty-five percent indicated that they would achieve their learning goals somewhat more effectively with such a learning environment. The usefulness of the learning environment was rated diversity. 


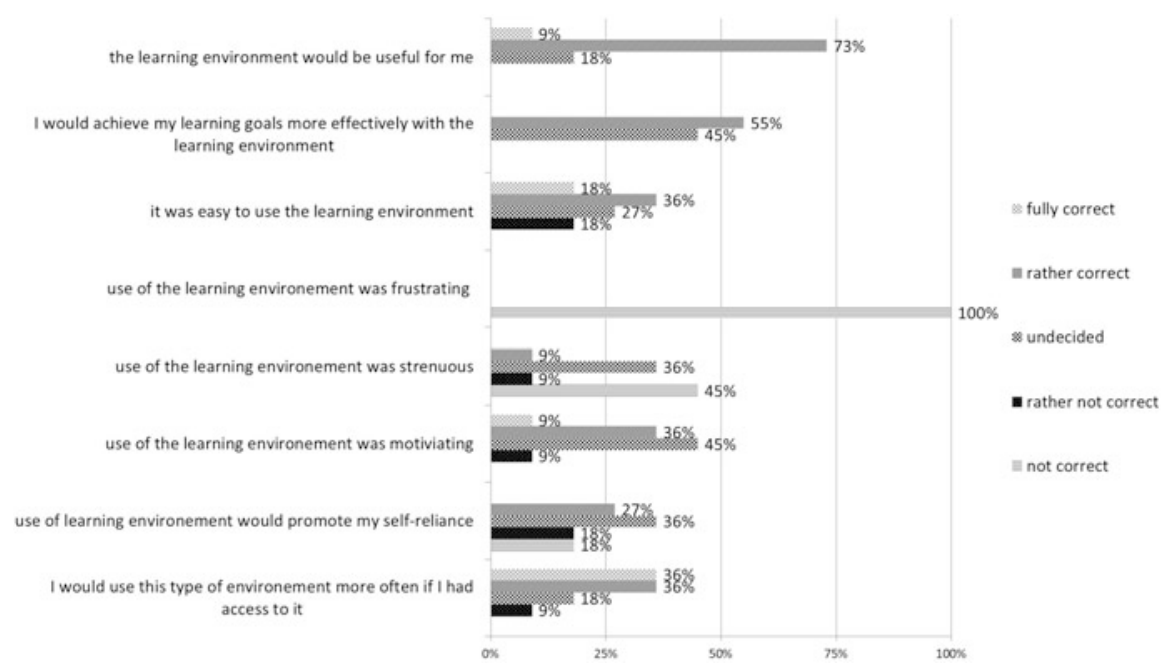

Fig. 6 Results of the questionnaire "Perceived Usefulness and Ease of Use"

Eighteen percent chose "fully correct" and $36 \%$ rather correct while $27 \%$ were undecided and $18 \%$ said that the uses were rather not easy.

There was a strong agreement with $100 \%$ that the use of the learning environment was not frustrating. But the evaluation showed that there was still a need for improvement. Almost half of the interviewees (45\%) said the use of the learning environment was strenuous or rather strenuous. However, the vast majority with $72 \%$ of the respondents would use or rather use the learning environment, while only $9 \%$ said that they rather not use the tested learning technology. The results in details were shown in the Fig. 6 .

Further, through the use of the "TAM" it was possible to document positive as well as negative statements of the probands. Figure 7 represents a compendium of the most important statements, divided into the categories effort, performance and facilitation.

In conclusion, the evaluation results of the PLMS allowed the following statements:

- The use of the PLMS is deemed highly beneficial

- The PLMS supports the achievement of individual learning goals

- The PLMS would be used by the learners, if access to a tool was available

- Usability, as well as the look and feel of the PLMS prototypes, must be greatly improved

In terms of challenges, however, some technical hurdles appeared regarding the usability as well as look and feel of the PLMS for future implementations. These challenges related to specific computer-based issues and are all undergoing further investigation. 


\begin{tabular}{|c|c|c|}
\hline $\begin{array}{l}\text { How user-friendly are the PLMS and the } \\
\text { integrated widgets? } \\
\text { (effort) }\end{array}$ & $\begin{array}{l}\text { Are performance and functions } \\
\text { recognised and appreciated? } \\
\text { (performance) }\end{array}$ & $\begin{array}{l}\text { How well supported does the learner } \\
\text { feel within the learning environment? } \\
\text { (facilitation) }\end{array}$ \\
\hline $\begin{array}{l}\text { Positive: } \\
\text { - Hardly any problems adding the } \\
\text { widgets } \\
\text { Breakdown into steps promotes } \\
\text { clarity } \\
\text { Room for improvement: } \\
\text { - Confusion upon exiting the PLMS } \\
\text { and entering the browser } \\
\text { Usability of many of the widgets is } \\
\text { faulty } \\
\text { The monitor is frequently overloaded } \\
\text { with scroll bars }\end{array}$ & $\begin{array}{l}\text { Positive: } \\
\text { - Media lists facilitate research on } \\
\text { the Internet } \\
\text { Room for improvement: } \\
\text { - Creation of private media lists } \\
\text { would be desirable } \\
\text { Create an option for integrating } \\
\text { well-known tools } \\
\text { Conventional storage media are } \\
\text { given precedence }\end{array}$ & $\begin{array}{l}\text { Positive: } \\
\text { Room for improvement: } \\
\text { - Video for the "Learn" phase is } \\
\text { too long } \\
\text { Content of the wizard is not } \\
\text { understandable at first glance } \\
\text { Help questions, tips and } \\
\text { recommended tool categories do } \\
\text { not provide enough help } \\
\text { It's frequently unclear when the } \\
\text { individual learning phases are } \\
\text { completed }\end{array}$ \\
\hline
\end{tabular}

Fig. 7 Statements from the thinking aloud interviews

Nonetheless the feedback from the evaluation investigation remains very positive. It appears that people really liked the PLMS approach. Additionally, and since the test phases took place, albeit on a prototype, the interviewees asked also for refinements of the system in relation to their user experience.

\section{Conclusion and Outlook}

\section{The Future of TEL in Business Context}

The ROLE approach and the gained experience showed the potential of TEL solutions not only in the higher education field, but also in business context. This sounds quite simple at first, but it is extremely important. Higher education settings and learning at a company are not two different worlds, but completely different learning scenarios, with different learning goals and needs, different learners, different learning conditions and learning possibilities. Often in research projects initial solutions are presented without entirely knowing the specific surrounding conditions.

But these kinds of solutions are more innovative than useful. There are a lot of hurdles and specifications in companies according to organisational, technical and personnel requirements. Therefore, it is important for TEL researchers to start every TEL activity with a target group-specific requirement analysis and after this to transfer the acquired scientific developments into these new learning environments.

On the other hand, for bigger companies it is important to be open to new ideas, opportunities and learning approaches as that can help to identify, share and archive important knowledge in a better or additional way than before. Often a big revolution is not necessary. Companies have a lot of different learning technologies and learning possibilities that are, however, separate, internal closed systems without 
synchronisation and different responsible departments and responsible persons. Thus, these learning technologies are in internal and in external competition with one another and a systematic integration of technology-enhanced learning tools is a challenge.

During the ROLE project, Festo has used its chance to learn from the ROLEconsortium and bring new research results together with specific business requirements. During the project the Virtual Academy was opened up through enhancing CLIX by OpenSocial widgets. The different evaluation loops showed that is not only a current trend move away from closed LMS to more self-regulated and individualised learning. Rather, it confirmed the first impression of the project team to combine the advantages of the existing LMS with the PLE approach. One outcome of this approach was the Festo LearningTube which enables every Festo employee to produce and share user (expert) generated learning videos on the LMS.

The good thing is not only the received Comenius award but also the fact that it is possible to share very easily user generated content in a company now. The knowledge carriers produce their content on a voluntary basis without the necessity of additional incentives. This supported Festo significantly in improving this learning environment with the developed technologies and the intelligent combination of formal and informal learning.

\section{What Are the Next Steps?}

The Virtual Academy, especially the LearningTube will be continuously improved. In this context three topics will be more and more important:

- Learning analytics

- e-Learning goes mobile

- Full text search in videos

Learning analytics is a very interesting field for Festo in terms of learning transfer analysis. Researching how this process can be taken beyond the step of e-tests and e-evaluation is a specific interest of Festo. Just in time analysis of user data, visualising learning processes to users, context aware services and individual recommendations based on learning goals or learning needs would be one of the future scenarios.

When Festo started with the Virtual Academy it was only accessible via the Intranet, so people could only learn from their workplaces. This was considered as a bottleneck for the learning processes, so the platform was moved on a server that is also accessible via the Internet. The goal is to make use of the benefits of e-learning especially learning independent from time and location. This step, taken in 2005 , can be taken even beyond by bringing content also to mobile devices such as mobile phones and tablets. This opportunity should be realised as soon as possible, especially because the demand of the learners for mobile learning services is continuously growing. A big lever for this approach is that the videos from the 
LearningTube are mobile compliant and can therefore be easily "consumed" from mobile devices.

Video-based learning is more and more an essential source of knowledge and information in companies. As described before, the Festo LearningTube is a rapidly growing internal video portal. At present there are more than 220 expert generated videos and more are being produced every day. Unfortunately the search function is not yet as developed. The growing number of videos results in a growing need for state of the art search functionalities to support learners as much as possible find the learning content they need. The objective is to find a solution that efficiently combines voice recognition with the videos on the Virtual Academy and make them thereby "full text searchable".

\section{Conclusion}

The ROLE philosophy of Festo was to open the existing Virtual Academy LMS and extend it with new technologies supporting individualisation of the learning process and increasing its efficiency. The LMS and PLE approaches were combined towards a PLMS fulfilling existing business requirements. This was technically realised by means of integration of the PLE elements into the LMS platform and pedagogically with the use of SRL method. As previously described, the first step was to implement two widgets (media search and media list widgets) in the existing Festo Virtual Academy LMS. At the second step, this approach was extended and realised as a PLMS supporting four learning phases of the SRL process (plansearch-learn-reflect).

The use of the PLMS at workplace supports, on the one hand, curriculum-based learning allowing completing learning programmes and courses created by an organisation for its employees. On the other hand, it extends usual personnel development measures with SRL activities giving the employees an opportunity to specialise in the most important and interesting fields of knowledge, thus, developing their competencies and skills in an individualised manner addressing personal strengths and preferences. This assures gaining of obligatory knowledge and skills for completing specific tasks by the employees and supports personal development, thus removing borders between workplace and spare time learning.

Moreover, while using the PLMS SRL competencies, such as goal setting, planning, time management, resources search, and self-evaluation, are trained. These skills can be applied by the learners not only in training context but also at the workplace helping to improve every-day working processes and achieving better results. Festo sees the need to support learners with the new technology and to develop SRL and media competence as key factors for successful working with the PLMS and the ROLE technologies. In this development process Festo keeps in mind that, in a business context, there are complex requirements and restrictions, for example, the contrasts between: 
- Openness versus data security (e.g. the work council, the data protection officer and some others had very different opinions and views on the proposed work)

- Companies' targets versus individual targets

- Implementation strategy: the "Revolution" (completely new technology) versus the "Evolution" (successive further development of existing tools)

The conclusion, therefore, is that the current vision and deployment of a PLE towards an integrated PLMS implementation with predefined learning spaces on the technical side seems to have been warmly welcomed. Nonetheless, from the psycho-pedagogical perspective it remains essential that learners are able to learn in a self-regulated manner.

The required technical improvements, therefore, have to be synchronised with the necessary individual development of specific SRL competences in order to meet these very real needs. The bigger pedagogical challenge, however, will be to promote the new approach to learners on a large scale. The evidence that this is possible is the fact that the LearningTube widget bundle has become an essential part of the Virtual Academy.

Open Access This chapter is distributed under the terms of the Creative Commons Attribution Noncommercial License, which permits any noncommercial use, distribution, and reproduction in any medium, provided the original author(s) and source are credited.

\section{References}

Attwell G. The Personal Learning Environments-the future of eLearning? eLearning Papers. 2007;2(1). http://www.elearningeuropa.info/files/media/media11561.pdf

Fruhmann K, Nussbaumer A, Albert D. A psycho-pedagogical framework for self-regulated learning in a responsive open learning environment. In: Hambach S, Martens A, Urban B, editors. Proceedings of the 3rd International eLBa Science Conference, Rostock, 2010.

Lewin T. Instruction for masses knocks down campus walls. The New York Times, March 4, 2012. http://www.nytimes.com/2012/03/05/education/moocs-large-courses-open-to-all-topple-campuswalls.html

Mödritscher F, Nussbaumer A. Self-regulated learning-communities-of-practice vs. selfregulated learning - motivation, backgrounds, and modus operandi of this blog. In: COPSRL, Nov 23, 2012. http://augur.wu-wien.ac.at/COPSRL/?cat $=5$

Overton L. An Introduction to personal learning environments. Making learning personal—using PLEs to enhance learning. In: Towards maturity, Nov 18, 2009. http://www.towardsmaturity. org/article/2009/11/18/introduction-personal-learning-environments/

Schanda F, Dikke D, Mueller N. Personal learning management systems (PLMS): concept, classification, evaluation. In: eLearning Baltics 2012. Proceedings of the 5th International eLBa Science Conference in Rostock, Germany, June 21-22, 2012. 